\title{
EPILEPTIC SEIZURE DETECTION USING BRIAN SIGNAL PROCESSING
}

\begin{tabular}{|l|l|l|}
\hline Syed Umar Farooq & Mallikarjun H M & Dr. H N Suresh \\
MTech, Industrial Electronics, RNSIT, & Asst Prof, Dept of E \& I, RNSIT, & Prof, Dept of E \& I, BIT, Bangalore, \\
Bangalore & Bangalore & hn.suresh@ @ediffmail.com \\
syedumarplus@gmail.com & Research Scholar, ECE Dept, Karpagam & \\
& $\begin{array}{l}\text { University-CBT } \\
\text { mallikarjunhm@ymail.com }\end{array}$ & \\
\hline
\end{tabular}

Abstract - This work proposes a method of detection of epileptic seizure using Wavelet Transform and wavelet based features. Linear classifier has been used to classify Normal and epileptic Electroencephalogram (EEG) signals. For seizure detection, the database collected from Spandana Hospitals Pvt. Ltd, Bangalore has been used. Ten EEG signals were recorded from healthy volunteer with eye open, and ten EEG signals were recorded from epilepsy patients during epileptic seizures. Important features such as standard deviation, entropy, maximum, mean and minimum at different sub bands were calculated and classification was done using Adaptive NeuroFuzzy Interface System (ANFIS) classifier tool and an error $6.5773 \mathrm{e}^{-05}$ was observed.

Keywords-EEG, CWT, DT, ANFIS, EDF

\section{INTRODUCTION}

One of the most prevalent neurological disorders that we can find in children and adults is Epilepsy. It is characterized by recurring of seizures which causes the loss of consciousness due to the abnormal electrical activity in the brain. People may suffer from physical injury as they are usually unaware of the appearance of seizure due to its random nature. Studies show that $4-5 \%$ of the total world population is suffering from epilepsy [1]. Electroencephalogram (EEG) is one of the important tool available for the detection and diagnosis of epilepsy. Electroencephalogram is a record of the electric signals generated by the synchronous (occurring at the same time) action of brain cells. For recording of EEG, electrodes will be placed at some key points on the patient's scalp. Recorded signals will be used for future diagnosis. Electrodes picks up the electrical signals generated by human brain and will be recorded in a memory device through several wires which are connected to the respective electrodes. To apply the location of electrodes for EEG recording the "10-20" system is used which is an internationally accepted method. In the " $10-20$ " system the actual distances between electrodes is $10 \%$ or $20 \%$ of front-back / right-left distance of the scalp [2-4].

A complete visual examination of an EEG signal is very arduous. However as EEG signal is a non-stationary signal, Fourier analysis does not provide accurate results. Most constructive time-frequency analysis tool for the analysis of transient signals is wavelet transform analysis. [5$10]$
The diagnosis of epilepsy can be subdivided into following modules i. Preprocessing, ii. Feature extraction, and iii. Classification of Seizure detection [11-16]. Research work on seizure detection began in 1980's. Gotman proposed a remarkable work on epilepsy detection in the year 1982. [5]. Method for classification of epileptic and no epileptic data by using wavelets was proposed by Khan and Gotman.

In 2005 short time Fourier transform method was compared with wavelet transform method to obtain the best model in order to get an overall good accuracy in determining the epilepsy EEG signals obtained from human brain. Wavelet based combinational model for the classification of features from EEG signals was first suggested by Ubeyli. Important features such as entropy, energy, and standard deviation at different sub bands were computed using wavelet decomposition and Windowed Fourier Transform. Feature vectors were used to test and train the neural network algorithm toolbox and classification accuracies were obtained by evaluating the algorithm by using suitable MATLAB command for each of the wavelet families.

\section{FUNCTION MODEL}

The Electroencephalogram (EEG) signals are obtained from real-time database. EEG signals are processed in MATLAB. This can be useful in classification of different disorders using classifier tools present in it. The features are extracted from frequency bands (alpha, delta and theta). Block diagram for Epileptic Seizure Detection using brain signal processing shown in the Fig 1.

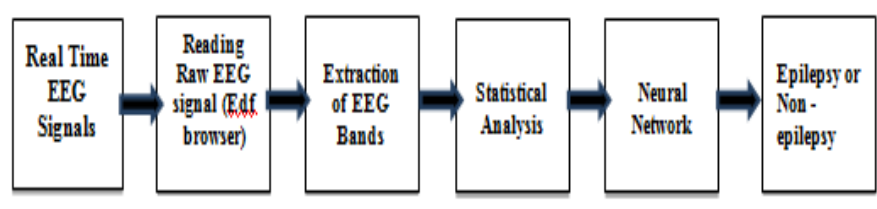

Fig. 1. Block Diagram of the proposed system

\section{MATERIALS AND METHODS}

\section{A. EEG Database}

Real time EEG Data base was used for the detection of epilepsy. Database was obtained from Spandana Hospital Pvt. 


\section{Asia Pacific Journals}

Ltd. The recording was obtained by using standard 10-20 electrode system. Twenty EEG recordings of which 10 EEG signals were recorded from healthy volunteer with eye open, and another 10 EEG signals were recorded from epilepsy patients during epileptic seizures. 10-20 electrode placement system was used. The Mastoids were used as references. The data was originally sampled at a frequency of $256 \mathrm{~Hz}$, and filtered 20 from $0.05-100 \mathrm{~Hz}$. Data were then down sampled at $128 \mathrm{~Hz}$ and they stored in .mat files.

\section{B. Wavelet Transform}

Wavelet transform is a series representation of time function with respect to fixed building blocks, simply termed as wavelets. Using translation and dilation operations of single generating function called mother wavelets these derived building blocks are a family of functions. The advantage of WT is that it has enlarged window size; it is narrow at high frequency and broad at low frequency. In all frequency ranges it leads towards an optimal time - frequency resolution. EEG signals consisting of many sampling points can be compressed into few features by performing spectral analysis using wavelet transform.

The wavelet transform can be categorized into discrete and continuous types. Continuous wavelet transform is defined as

$$
C W T(a, b)=\int_{-\infty}^{\infty} x(t) \varphi_{a, b}^{*}(t) d t
$$

Where $\mathrm{x}(\mathrm{t})$ be entitled as the analyzing signal and $a$ is entitled as the scaling factor (dilation/compression coefficient) and $b$ is entitled as the translation along time axis (shifting coefficient).The superscript asterisk indicates the complex conjugation $(a, b)($.$) is computed by scaling the$ wavelet at time $b$ and scale $a$ :

$$
\varphi_{a, b}(t)=\frac{1}{\sqrt{|a|}} \varphi\left(\frac{t-b}{a}\right)
$$

Where implies the wavelet. In continuous WT, the translation and scaling parameters " $a$ " and " $b$ " can be changed in a continuous manner. However, computing wavelet coefficients for every possible scale can result in a huge quantity of data. Therefore discrete wavelet transform (DWT) is commonly used. This can be assumed as an extension of classic Fourier transform method. Instead of working on a single scale (frequency or time), it works on a multi scale basis. Decomposition of a Multi-resolution signal $\mathrm{x}(\mathrm{n})$ is schematically shown in Fig2.
ISBN: 978-81-930411-5-4

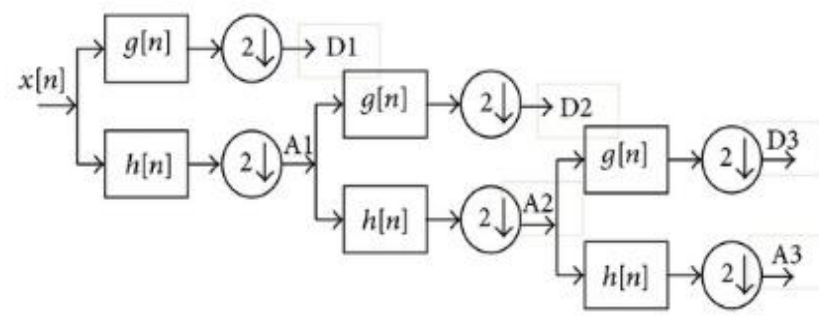

Fig. 2. Sub-band decomposition of DCT implementation.

Each stage of this model consists of 2 down samplers and two digital filters. The first filter $\mathrm{g}($.) is of high pass in nature and the second $\mathrm{h}($.$) is its mirror version which is of low pass$ in nature. The down sampled outputs of first low-pass and high- pass filters provides the approximation A1 and detail D1 respectively. A1 (first approximation) undergoes further decomposition and this decomposition is continued up to the required level shown in Fig 2.

\section{Parameters for Feature Extraction}

The EEG signals contain numerous data points; this can be minimized / compressed into few features that can be used to differentiate between different classes. The features that are used include some statistical features without wavelet decomposition and some wavelet based features.

Wavelet Based Features. The parameters that can be used after the wavelet decomposition process are Standard deviation, Entropy, Energy, Maximum and Minimum.

$\mathrm{EDi}=\sum_{j=1}^{N}\left|D_{I J}\right|^{2}, i=1,2,3, \ldots \ldots, p$,

$$
E A_{i}=\sum_{j=1}^{N}\left|A_{I J}\right|^{2}
$$

The energy at each decomposition level is calculated as

$$
E N T_{i}=\sum_{j=1}^{N} D_{I J}^{2} \log \left(D_{I J}^{2}\right), \mathrm{i}=1,2,3, .
$$

The entropy at all decomposition level has to be computed as where, $\mathrm{i}=1,2,3, \ldots, \mathrm{p}$ is wavelet decomposition level from 1 to $\mathrm{p}$ and it indicates the number of approximation or detail at each of the decomposition level.

The standard deviation for decomposition level was computed by using the following equation:

$$
\sigma_{i}=\left(\frac{1}{N-1} \sum_{j=1}^{N}\left(D_{i j}-\mu_{i}\right)^{2}\right)^{\frac{1}{2}},
$$

Where is the mean and is given by 


$$
\mu_{i}=\frac{1}{N} \sum_{j=1}^{N} D_{i j}, i=1,2,3, \ldots \ldots, l,
$$

\section{Classifier: Adaptive Neuro-Fuzzy Interface System (ANFIS)}

ANFIS belongs to the class of rules extracting systems using a de-compositional strategy, where rules are extracted at the level of individual nodes within the neural network and then aggregates these rules to form global behaviour descriptions. The training matrix is given as input to the editor of ANFIS. Using a given input/output data matrix, the toolbox function 'Anfis' constructs a FIS (fuzzy inference system) whose membership function parameters are adjusted using either a back propagation algorithm lone, or in combination with a least squares method. This makes your fuzzy system to learn from the data matrix they model. ANFIS depends on the sizes of the training set and testing set. ANFIS is implemented with MATLAB software. Features extracted from MATLAB code is given as input to ANFIS. Classification is carried out in two steps using ANFIS: Training and Testing.

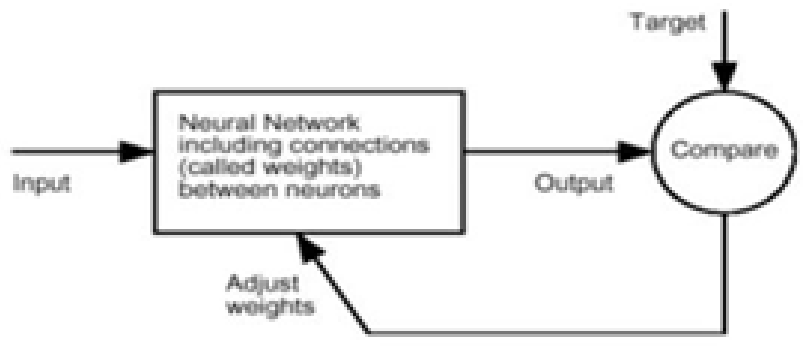

Fig. 3. Neural network block diagram

\section{RESUlTS AND DISCUSSION}

Database was obtained from Spandana Hospital Pvt. Ltd. The standard 10-20 electrode placement system was used for recording. The data used has gone through the preprocessing steps so that extraction of parameters becomes easy.
A. Feature Extraction Using Discrete Wavelet Transform. For the classification and analysis of EEG signals the number of decomposition and levels appropriate wavelet were selected as they are very important for Using Discrete Wavelet Transform. The level of decomposition is having been chosen as the best so that the frequencies that are required for classification of the signal are stored in the wavelet coefficients.

B. Classification Using ANFIS. A classifier tool has been trained in such a way that it outputs a ' 0 ' for abnormal EEG, ' 1 ' for normal EEG. The extracted features were applied to this classifier. All 20 Data samples collected from all 20 patients were used for training and testing the Adaptive Neuro-Fuzzy Interface System. Following procedure was used for giving calculated features to the classifier.

Form the Input matrix " $A^{c e}$ in MATLAB for values of mean $^{\mu(i)}$ and standard deviation $\sigma(i)$ data obtained from extracted eeg signal.

In the "A" matrix the first 2 columns represent the extracted values (inputs), last column represents the output. The two columns represent the mean and standard deviation extracted from the signal respectively.

The network is trained with the corresponding training matrices for different epilepsy EEG signals. During training the training error is observed and the corresponding changes in the input parameters of the ANFIS model are made to reduce these errors. Window obtained after training is shown in the Fig 4. And after testing the classifier is tested for output. The testing output is shown in the Fig 5; Fig. 6 shows Resulting ANFIS structure.

Once the result is satisfactory, performance of the obtained FIS is tested.

By using the command "evalfis" in MATLAB the output obtained for the matrix is verified and $100 \%$ classifier accuracy is obtained.

Resulting ANFIS structure for the classifier is shown in Fig 6 This ANFIS structure has five layers which are input, input membership, rules, output membership and output.

Table 1. EEG Database used

\begin{tabular}{|l|l|l|}
\hline Patient No & Sex & Age(Years) \\
\hline 01 & M & 44 \\
\hline 02 & M & 28 \\
\hline 03 & M & 31 \\
\hline 04 & M & 16 \\
\hline 05 & M & 16 \\
\hline 06 & F & 12 \\
\hline
\end{tabular}




\section{Asia Pacific Journals}

\begin{tabular}{|l|l|l|}
\hline 07 & M & 07 \\
\hline 08 & M & 19 \\
\hline 09 & M & 17 \\
\hline 10 & M & 27 \\
\hline 11 & F & 45 \\
\hline 12 & F & 54 \\
\hline 13 & F & 39 \\
\hline 14 & F & 23 \\
\hline 15 & F & 55 \\
\hline 16 & M & 77 \\
\hline 17 & M & 28 \\
\hline 18 & M & 13 \\
\hline 19 & F & 22 \\
\hline 20 & M & 30 \\
\hline
\end{tabular}

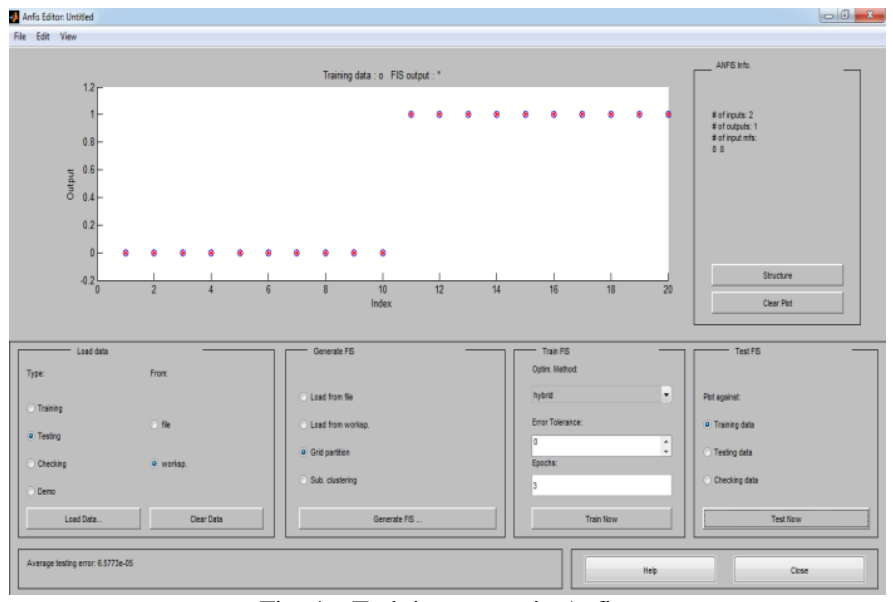

Fig. 4. Training output in Anfis

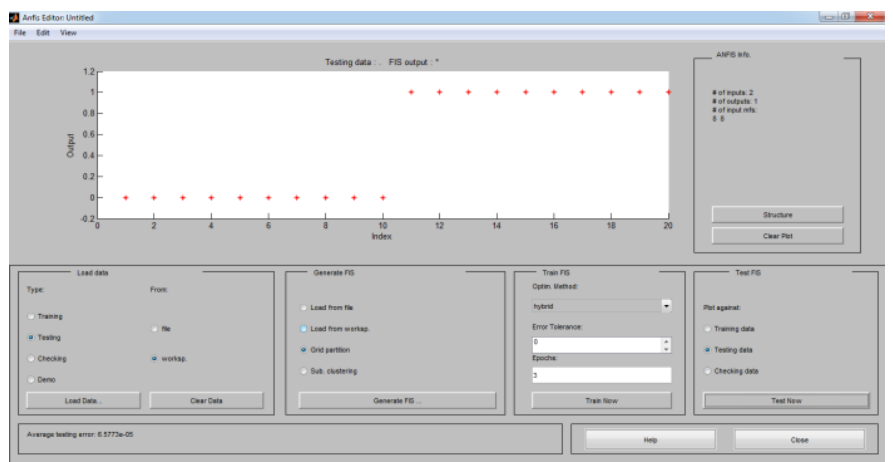

Fig. 5. Testing output in Anfis

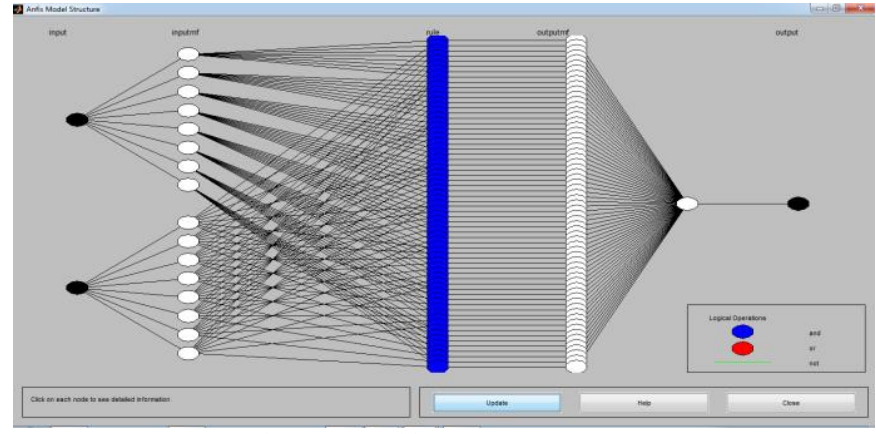

Fig. 6. Resulting ANFIS structure

\section{CONCLUSION}

In this work we developed a classifying model for the epileptic seizures. Real time EEG signals were used and features such as mean, standard deviation, energy, maximum, minimum and entropy values were calculated by using wavelet transform. Adaptive Neuro-Fuzzy Interface System has been used as classifier tool and an error of $6.5773 \mathrm{e}-05$ was observed. A total of $100 \%$ classification accuracy has been attained.

\section{FUTURESCOPE}

Epileptic seizure is a medical challenge and hence prediction plays vital role to prevent its side effects. This work may be extended by increasing number of datasets to make highly reliable predictor module. In future work may be carried by using EEG Brain CAP to acquired real time brain wave. Work may extended by adding advanced classification rules on the fuzzy sets and by using advanced $\mathrm{NN}$ algorithms.

\section{ACKNOWLEDGMENT}

The Authors would like to thank the management of R N S Institute of Technology- Principal Dr. M K Venkatesha, Director Dr. H N Shivashankar for the encouragement. Authors extend special thanks to Spandana Hospitals Pvt, Bangalore for sharing EEG Database.

\section{REFERENCES}

[1] "Automatic detection of seizure onset in pediatric EEG," Y. U. Khan, P. Sharma, and O. Farooq, IJE SA, vol. 2, no. 3, pp. 81-89, 2012.

[2] "Statistics over features: EEG signals analysis," E. D. " Ubeyli, CBM vol. 39, no. 8, pp. 733-741, 2009.

[3] "Epileptic Seizure Detection in Grouped Multi-Channel EEG Signal Using ICA and Wavelet Transform", Department of Neurology Kaohsiung Medical University Hospital Kaohsiung, Taiwan

[4] "Wireless instrumentation system based on dry electrodes for acquiring EEG Signals", NunoSérgioDiasa,b, João Paulo Carmoc,*, Paulo MateusMendesc, José HiginoCorreiaca Life and Health Sciences Research Institute (ICVS), School of Health Sciences, University of Minho, Braga,Portugalb ICVS/3B's- PT Government Associate Laboratory, Braga/Guimarães, Portugalc University of Minho, Dept Industrial Electronics, Campus Azurem, 4800-058, Portugal 


\section{Asia Pacific Journals}

[5] "EEG Signal Analysis: A Survey", D. Puthankattil Subha\& Paul K. Joseph \& Rajendr acharya U \& Choo Min Lim, Received: 25 August 2008 / Accepted: 29 October 2008

[6] "A micro-power EEG acquisition SoC with integrated feature extraction processor for a chronic seizure detection system", N Verma, A Shoeb, J Bohorquez, J Dawson, J Guttag, AP Chandrakasan SolidState Circuits, IEEE Journal of 45 (4), 804-816

[7] "Discrete Wavelet Transform Based Classification of human Emotions Using Electroencephalogram Signals", Mohamed Rizon. American Journal of Applied Sciences 7 (7): 878-885, 2010.

[8] "Combined neural network model employing wavelet coefficients for EEG signals classification." Übeyli, E.D.: Digital Signal Processing 19, 297-308 (2009)

[9] "Wavelet based feature for Epileptic Seizure Detection,", O. Farooq, M. Bedeeuzzaman, T. Fathima and Y. U. Khan, MESJTM, vol. 2, no. 1, pp. 108-112, 2011.

[10] "Wavelet transform, time-frequency localization and signal analysis," I Daubechies, IEEE TIT, vol. 36, no. 5, pp. 961-1005, 1990.

[11] "Estimation of hypnosis susceptibility based on electroencephalogram signal features", Z. Elahi a, R. Boostani a, A. MotieNasrabadi ba Department of Electrical and Computer Engineering, Shiraz University, Shiraz, P.O. Box: 7134851154, Iranb Department of Electrical Engineering, Shahed University, Tehran, P.O. Box: 3319118651, IranReceived 4 May 2010; revised 13 November 2011; accepted 1 July

[12] "Epileptic seizure detection using AR model on EEG signals", SR Mousavi, M Niknazar, B V Vahdat Biomedical engineering conference, 2008. CIBEC 2008. Cairo International.

[13] "Wavelet based automatic seizure detection in intra cerebral electroencephalogram," Y. U. Khan and J. Gotman, Clinical Neurophysiology, vol. 114, no. 5, pp. 898-908, 2003.Computers in Biology \&Medicine, vol. 35, no. 7, pp. 603-616, 2005.

[14] "Estimation of hypnosis susceptibility based on electroencephalogram signal features", Z. Elahi a, R. Boostani a, A. MotieNasrabadi ba Department of Electrical and Computer Engineering, Shiraz University, Shiraz, P.O. Box: 7134851154, Iranb Department of Electrical Engineering, Shahed University, Tehran, P.O. Box: 3319118651, IranReceived 4 May 2010; revised 13 November 2011; accepted 1 July

[15] "The Relation between Eeg Prefrontal Asymmetry and Subjective Feelings of Mood Following 24 Hours of Sleep Deprivation Camila Ferreira", Andréa Deslandes, Helena Moraes, Maurício Cagy, Luiz Fernando Basile, Roberto Piedade, Pedro Ribeiro.

[16] "Continuous Wavelets Transformation the wavelets Implemented on a DSP chip for EEG monitoring", Authors: Chein-yue Chen, Ming-Da Ke, Cheng-Den Kuo Published: the 1st international Conference on Information Science and Engineering (ICISE 2009). 